\title{
FRACTIONAL BROWNIAN MODELS FOR VECTOR FIELD DATA
}

\author{
Pouya Dehghani Tafti, Member, IEEE, and Michael Unser, Fellow, IEEE \\ Laboratoire d'imagerie biomédicale, Ecole Polytechnique Fédérale de Lausanne (EPFL), \\ CH-1015 Lausanne, Switzerland
}

\begin{abstract}
In this note we introduce a vector generalization of fractional Brownian motion. Our definition takes into account directional properties of vector fields—such as divergence, rotational behaviour, and interactions with coordinate transformations-that have no counterpart in the scalar setting. Apart from the Hurst exponent which dictates the scale-dependent structure of the field, additional parameters of the new model control the balance between solenoidal and irrotational behaviour. This level of versatility makes these random fields potentially interesting candidates for the stochastic modelling of physical phenomena in various fields of application such as fluid dynamics, field theory, and medical image processing.
\end{abstract}

Index Terms-Fractional Brownian motion, fractional Brownian vector fields, stochastic modelling, vector fields, invariance, generalized random processes, characteristic functional.

\section{INTRODUCTION}

Although the processes we know today by the name of fractional Brownian motion ( $\mathrm{fBm}$ ) had already appeared in mathematical literature in the 40s (see for instance Kolmogorov [1]), it was after their systematic study by Mandelbrot and Van Ness in their 1968 paper [2]from where they obtained their current name-that they gained widespread popularity as models for self-similar stochastic phenomena and found applications in an increasing number of disciplines such as network traffic analysis, the study of financial markets, hydrology, and image processing, to name only a few [3-9].

The definition of $\mathrm{fBm}$ given by Mandelbrot and Van Ness is in 1D and involves a fractional integral with respect to the differential of Brownian motion. To extend the definition to multiple dimensions, it has been found expedient instead to characterize the random field axiomatically, à la Paul Lévy [10], as a Gaussian random field with zero mean that passes through the origin at $\boldsymbol{x}=\mathbf{0}$ almost surely and whose (stationary) increments obey the following condition on their variances

$$
\mathbb{E}\left\{\left|B_{H}(\boldsymbol{x})-B_{H}(\boldsymbol{y})\right|^{2}\right\}=c\|\boldsymbol{x}-\boldsymbol{y}\|^{2 H}
$$

This work was supported by the Swiss National Science Foundation under Grant 200020-121763. (in the case of $H=1 / 2$ one recovers the definition of standard Brownian motion with $d$ parameters) [11]. The parameter $H$ is the well-known Hurst exponent which ranges between 0 and 1 (exclusive).

The practical interest in fBm models is rooted in their remarkable quality of statistical self-similarity (that is, self-similarity in law), which goes hand-in-hand with the empirical observation of inverse power-law (or $1 / f^{2 H+d}$ ) power spectra in a wide range of applications; although the latter statement has to be made with caution, as the classical notion of power spectrum is not applicable in the case of fBm's, which are non-stationary.

To put the spectral behaviour of the field in evidence and on a rigorous mathematical foundation, it is helpful to give yet another characterization of the random field, this time in terms of a whitening operator. One may in fact define $\mathrm{fBm}$ as the solution of a fractional stochastic PDE with suitable boundary conditions (zero at $\boldsymbol{x}=\mathbf{0}$ up to a certain order); to wit, for $H \in(0,1), B_{H}$ is proved $[12,13]$ to be the solution of the fractional Poisson equation

$$
(-\Delta)^{\frac{H}{2}+\frac{d}{4}} B_{H}=W
$$

subject to $B_{H}(\mathbf{0})=0$ (a.s.), where $W$ is a white Gaussian noise field in the sense of generalized random processes [14]. The precise Fourier domain interpretation of the above equation makes use of the characteristic functional of $B_{H}$, which is related to its probability measure by the Bochner-Minlos theorem [15] (see also Tafti $\mathcal{E}$ al. [12] for a discussion of the connection between the above characterization and the harmonizable representation [16] of $\mathrm{fBm}$ ). In addition to emphasizing the spectral character of the random field (the whitening operator $(-\Delta)^{\frac{H}{2}+\frac{d}{4}}$ is represented by the power-law symbol $\left.\|\boldsymbol{\omega}\|^{H+\frac{d}{2}}\right)$, the above definition lends itself to a natural and direct generalization to the case of $H>1$, leading to Gaussian fields with stationary $n$ th-order increments [17]. A different but interesting generalization is to replace the Gaussian white noise field in (1) by a non-Gaussian white noise (such as a Poisson noise).

Our aim in the present note is to follow the above line of argument in order to define vector-valued random fields that are intended to serve as stochastic models 
for self-similar (i.e. fractal) physical vector quantities. As a consequence of the success of $\mathrm{fBm}$ models in the statistical study of images, motivation for such a generalization is partly derived from the progress in recent years of imaging modalities for vector-field measurement (such as Doppler ultrasound imaging and velocity mapping via time-resolved magnetic resonance imaging (MRI) [18]); however, it is also of significance that some important vector phenomena, such as turbulent flow, are understood to be inherently fractal [19].

Let us emphasize at this point that it is not with the trivial vector generalization of $\mathrm{fBm}$ (by means of concatenation of independent scalar fBm's in vector form) that we shall be primarily concerned here. Instead, throughout the presentation, we shall insist on certain directional properties of vector fields that have no counterpart in the scalar setting. The construction we propose puts at the forefront the invariance properties of the random field. These invariances single out a parametric family of operators $(-\boldsymbol{\Delta})_{\xi}^{\gamma}$ generalizing the fractional vector Laplacian with new parameters $\left(\xi_{1}, \xi_{2}\right)=\boldsymbol{\xi}$ that effectively control the balance between rotational and compressive behaviour of the field. Fractional Brownian vector fields are then defined as solutions of the equation

$$
(-\boldsymbol{\Delta})_{\boldsymbol{\xi}}^{\frac{H}{2}+\frac{d}{4}} \boldsymbol{B}_{H}=\epsilon_{H} \boldsymbol{W} .
$$

In what appears next, we first define the differential operators introduced above and describe their invariances (\$2). This is followed by the definition of vector fractional Brownian motion and a list of its important properties (§3). We then briefly mention how instances of such random fields can be simulated (\$4) and conclude with a recapitulation of the some of the key points (\$5).

\section{OPERATORS AND INVARIANCES}

We define our generalization of the fractional vector Laplacian as the operator $(-\Delta)_{\xi}^{\gamma}$ with Fourier expression

$$
\boldsymbol{\Phi}_{\boldsymbol{\xi}}^{\gamma}(\boldsymbol{\omega}):=\|\boldsymbol{\omega}\|^{2 \gamma}\left[\mathrm{e}^{\xi_{1}} \frac{\boldsymbol{\omega} \boldsymbol{\omega}^{\top}}{\|\boldsymbol{\omega}\|^{2}}+\mathrm{e}^{\xi_{2}}\left(\mathbf{I}-\frac{\boldsymbol{\omega} \boldsymbol{\omega}^{\top}}{\|\boldsymbol{\omega}\|^{2}}\right)\right]
$$

with $\gamma \in \mathbb{R}_{+}$and $\boldsymbol{\xi}=\left(\xi_{1}, \xi_{2}\right) \in \mathbb{R}^{2}$. This definition is guided by the invariance properties we wish the operator (and consequently the random vector fields to be defined) to possess, as detailed below [20].

\subsection{Invariance properties}

In formulating invariance properties we take our inspiration from two sources. The first is the quality of self-similarity or fractality. The second is the mathematical framework that governs the interaction of vector fields with coordinate transformations. The mathematical manifestation of these invariances is through the observation that the operator commutes with them.

\subsubsection{Self-similarity}

Self-similarity dictates that $(-\boldsymbol{\Delta})_{\xi}^{\gamma}$ should commute with scaling (up to a homogeneous scale factor), meaning that one should be able to interchange the order of application of $(-\boldsymbol{\Delta})_{\xi}^{\gamma}$ and a uniform scaling of the domain. This property holds in the following way (a Fourier domain verification of the result is straightforward).

$$
(-\boldsymbol{\Delta})_{\boldsymbol{\xi}}^{\gamma} \mathbf{S}_{\sigma}=\sigma^{2 \gamma} \mathbf{S}_{\sigma}(-\boldsymbol{\Delta})_{\boldsymbol{\xi}}^{\gamma}
$$

where $\mathbf{S}_{\sigma}: f(\boldsymbol{x}) \mapsto f\left(\sigma^{-1} \boldsymbol{x}\right)$ is the scaling operator.

\subsubsection{Vector rotation}

An elementary result of vector calculus states the following: Given two $\mathbb{R}^{d} \rightarrow \mathbb{R}^{d}$ representations of a vector field, one in the standard Cartesian coordinates $\boldsymbol{u} \in \mathbb{R}^{d}$ as $\boldsymbol{f}(\boldsymbol{u})$, and the second in rotated coordinates $\boldsymbol{x}=\boldsymbol{\Omega} \boldsymbol{u}$ as $f_{\Omega}(\boldsymbol{x})$ (where $\Omega$ is a rotation matrix), the representations are related by

$$
f_{\Omega}(x)=\boldsymbol{\Omega} f(u)=\boldsymbol{\Omega} \boldsymbol{f}\left(\boldsymbol{\Omega}^{\top} \boldsymbol{x}\right) .
$$

This motivates the definition of the vector rotation operator $\mathbf{R}_{\boldsymbol{\Omega}}: \boldsymbol{f}(\boldsymbol{x}) \mapsto \boldsymbol{\Omega} \boldsymbol{f}\left(\boldsymbol{\Omega}^{\top} \boldsymbol{x}\right)$.

The generalized Laplacian $(-\Delta)_{\xi}^{\gamma}$ commutes with $\mathbf{R}_{\Omega}$ for all rotation (and reflection) matrices $\Omega$. In other words, for any orthogonal matrix $\Omega$,

$$
(-\boldsymbol{\Delta})_{\xi}^{\gamma} \mathbf{R}_{\Omega}=\mathbf{R}_{\Omega}(-\boldsymbol{\Delta})_{\boldsymbol{\xi}}^{\gamma}
$$

2.2. Other properties of $(-\Delta)_{\xi}^{\gamma}$

$(-\boldsymbol{\Delta})_{\xi}^{\gamma}$ has these additional properties:

$$
\begin{array}{cc}
(-\boldsymbol{\Delta})_{\boldsymbol{\xi}_{1}}^{\gamma_{1}}(-\boldsymbol{\Delta})_{\boldsymbol{\xi}_{2}}^{\gamma_{2}}=(-\boldsymbol{\Delta})_{\boldsymbol{\xi}_{1}+\boldsymbol{\xi}_{2}}^{\gamma_{1}+\gamma_{2}} ; & \text { (composition) } \\
(-\boldsymbol{\Delta})_{\boldsymbol{\xi}}^{\gamma}=(-\boldsymbol{\Delta})_{\mathbf{0}}^{\gamma}\left[\mathrm{e}^{\xi_{1}} \mathbf{E}+\mathrm{e}^{\xi_{2}}(\mathrm{Id}-\mathbf{E})\right] ; & \text { (factorization) }
\end{array}
$$

where $\mathbf{E}$ is the operator corresponding to the (matrix) Fourier expression $\boldsymbol{\omega} \boldsymbol{\omega}^{\top} /\|\boldsymbol{\omega}\|^{2}$. The significance of the latter property lies in the observation that $\mathbf{E}$ projects a vector field onto its curl-free component, while (Id $-\mathbf{E})$ is a projection onto the divergence-free component. As a consequence, by adjusting the parameters $\xi_{1}$ and $\xi_{2}$, one can have control over the irrotational vs solenoidal character of the solution of (6).

\subsection{Inverse operators}

Since $(-\boldsymbol{\Delta})_{\xi}^{\gamma}$ has a non-trivial null-space (for instance, it eliminates constants and polynomials of low degree), it can be inverted from the right up to an arbitrary additive term from the null-space. A right inverse operator of particular interest is the one defined by the $d$-dimensional integral (using multi-index notation)

$$
\begin{aligned}
& (-\dot{\Delta})_{-\boldsymbol{\xi}}^{-\gamma} \boldsymbol{f}:=\frac{1}{(2 \pi)^{d}} \\
& \cdot \int_{\mathbb{R}^{d}}\left(\mathrm{e}^{\mathrm{j}\langle\boldsymbol{x}, \boldsymbol{\omega}\rangle}-\sum_{|\boldsymbol{k}| \leq\left\lfloor 2 \gamma-\frac{d}{2}\right\rfloor} \frac{\mathrm{j}^{|\boldsymbol{k}|} \boldsymbol{x}^{\boldsymbol{k}} \boldsymbol{\omega}^{\boldsymbol{k}}}{\boldsymbol{k} !}\right) \boldsymbol{\Phi}_{-\boldsymbol{\xi}}^{-\gamma}(\boldsymbol{\omega}) \hat{\boldsymbol{f}}(\boldsymbol{\omega}) \mathrm{d} \boldsymbol{\omega} .
\end{aligned}
$$


Note that $\boldsymbol{\Phi}_{-\xi}^{-\gamma}(\boldsymbol{\omega})$ is the matrix inverse of $\boldsymbol{\Phi}_{\xi}^{\gamma}(\boldsymbol{\omega})$ (the Fourier symbol for $\left.(-\Delta)_{\xi}^{\gamma}\right)$ at all points except at $\boldsymbol{\omega}=\mathbf{0}$, and that the polynomial correction inside the integral is there to cancel the singularity and impose zero boundary conditions in the spatial domain at $\boldsymbol{x}=\mathbf{0}$. These boundary conditions are important for defining fractional Brownian motion vector fields.

Later, we shall also need the adjoint of $(-\dot{\Delta})_{-\xi}^{-\gamma}$, that is, the operator $(-\grave{\Delta})_{-\xi}^{-\gamma}$ satisfying

$$
\left\langle\phi,(-\dot{\Delta})_{-\xi}^{-\gamma} \psi\right\rangle=\left\langle(-\grave{\Delta})_{-\xi}^{-\gamma} \phi, \psi\right\rangle
$$

over $\mathcal{S}^{d}$. This operator (which we call the left inverse) is given by the relation

$$
\begin{aligned}
& (-\grave{\Delta})_{-\xi}^{-\gamma} \boldsymbol{f}:=\frac{1}{(2 \pi)^{d}} \\
& \quad \cdot \int_{\mathbb{R}^{d}} \mathrm{e}^{\mathrm{j}\langle\boldsymbol{x}, \boldsymbol{\omega}\rangle} \boldsymbol{\Phi}_{-\boldsymbol{\xi}}^{-\gamma}(\boldsymbol{\omega})\left(\hat{\boldsymbol{f}}(\boldsymbol{\omega})-\sum_{|\boldsymbol{k}| \leq\left\lfloor 2 \gamma-\frac{d}{2}\right\rfloor} \frac{\hat{\boldsymbol{f}}^{(\boldsymbol{k})}(\mathbf{0}) \boldsymbol{\omega}^{\boldsymbol{k}}}{\boldsymbol{k} !}\right) \mathrm{d} \boldsymbol{\omega} .
\end{aligned}
$$

The following observation is of key importance: $(-\grave{\Delta})_{-\xi}^{-\gamma}$ maps rapidly-decaying smooth test functions of Schwartz class (i.e. $f \in \mathcal{S}^{d}$ ) to functions with finite energy ( $L_{2}^{d}$ functions with $\|\boldsymbol{f}\|^{2}=\left\|\boldsymbol{f} ; L_{2}^{d}\right\|^{2}:=\sum_{i}\left\|f_{i} ; L_{2}\right\|^{2}$ ).

\section{FRACTIONAL BROWNIAN VECTOR FIELDS}

We define the normalized vector fractional Brownian motion with parameters $H$ and $\boldsymbol{\xi}=\left(\xi_{1}, \xi_{2}\right)$ as the selfsimilar random vector field that is whitened by the generalized fractional Laplacian $(-\boldsymbol{\Delta})_{\xi}^{\frac{H}{2}+\frac{d}{4}}$; that is,

$$
(-\boldsymbol{\Delta})_{\boldsymbol{\xi}}^{\frac{2 H+d}{4}} \boldsymbol{B}_{H, \boldsymbol{\xi}}=\epsilon_{H} \boldsymbol{W},
$$

with $\boldsymbol{W}$ a vector of normalized independent white Gaussian noise fields and the constant $\epsilon_{H}$ defined by the relation

$$
\epsilon_{H}^{2}=(2 \pi)^{\frac{d}{2}} \frac{2^{2 H+d / 2} \Gamma\left(H+\frac{d}{2}\right)}{|\Gamma(-H)|} .
$$

In addition, $\boldsymbol{B}_{H, \boldsymbol{\xi}}$ (and its appropriate derivatives in the extended case of $H>1$ ) are required to vanish almost surely at $\boldsymbol{x}=\mathbf{0}$.

A more direct statement of the definition can be made using the inverse operator introduced in the previous section:

$$
\boldsymbol{B}_{H, \boldsymbol{\xi}}:=\epsilon_{H}(-\dot{\boldsymbol{\Delta}})_{-\boldsymbol{\xi}}^{-\frac{2 H+d}{4}} \boldsymbol{W} .
$$

We should note that a careful application of an operator to a random field as in the above equation necessitates an appropriate mathematical formalism. Our way of procedure is based on Gel'fand and Vilenkin's theory of stochastic analysis using generalized functions; which is to say that $\boldsymbol{B}_{H, \boldsymbol{\xi}}$ is identified by means of a probability measure on Borel cylinder sets of the form

$$
\begin{array}{r}
\left\{\chi:\left\langle\chi, \phi_{1}\right\rangle<a_{1}, \ldots,\left\langle\chi, \phi_{N}\right\rangle<a_{N}\right\} \subset\left(\mathcal{S}^{d}\right)^{\prime}, \\
N \in \mathbb{N}, \phi_{1}, \ldots, \phi_{N} \in \mathcal{S}^{d} ;
\end{array}
$$

or, equivalently (by Minlos's version of Kolmogorov's extension theorem), via consistent finite dimensional distributions of its products $\left\langle\boldsymbol{B}_{H, \boldsymbol{\xi}}, \boldsymbol{\phi}\right\rangle$ with test functions $\phi$ (these products being classical real-valued random variables). By the Bochner-Minlos theorem [15], such probability measures on $\left(\mathcal{S}^{d}\right)^{\prime}$ are in one-to-one correspondence with continuous, positive-definite characteristic functionals defined as per

$$
Z_{\boldsymbol{B}_{H, \boldsymbol{\xi}}}(\boldsymbol{\phi}):=\mathbb{E}\left\{\mathrm{e}^{\mathrm{j}\left\langle\boldsymbol{B}_{H, \boldsymbol{\xi}}, \boldsymbol{\phi}\right\rangle}\right\}=\int_{\left(\mathcal{S}^{d}\right)^{\prime}} \mathrm{e}^{\mathrm{j}\langle\boldsymbol{\chi}, \boldsymbol{\phi}\rangle} P_{\boldsymbol{B}_{H, \boldsymbol{\xi}}}(\mathrm{d} \boldsymbol{\chi}) .
$$

We make use of duality and the known characteristic functional of the white Gaussian noise $\boldsymbol{W}$ to find the explicit form of the characteristic functional of $\boldsymbol{B}_{H, \boldsymbol{\xi}}$. The former functional is, (almost by definition)

$$
Z_{W}(\phi)=\mathrm{e}^{-\frac{1}{2}\|\phi\|^{2}} ;
$$

whence,

$$
\begin{array}{rlrl}
Z_{\boldsymbol{B}_{H, \boldsymbol{\xi}}}(\boldsymbol{\phi}) & =\mathbb{E}\left\{\mathrm{e}^{\mathrm{j}\left\langle\boldsymbol{B}_{H, \boldsymbol{\xi}}, \boldsymbol{\phi}\right\rangle}\right\} & \\
& =\mathbb{E}\left\{\mathrm{e}^{\mathrm{j} \epsilon_{H}\left\langle(-\grave{\Delta})_{-\boldsymbol{\xi}}^{-\frac{2 H+d}{4}} \boldsymbol{W}, \boldsymbol{\phi}\right\rangle}\right\} & & \text { by (7); } \\
& =\mathbb{E}\left\{\mathrm{e}^{\mathrm{j} \epsilon_{H}\left\langle\boldsymbol{W},(-\grave{\boldsymbol{\Delta}})_{-\boldsymbol{\xi}}^{-\frac{2 H+d}{4}} \phi\right\rangle}\right\} & & \text { by (4); } \\
& =Z_{\boldsymbol{W}}\left(\epsilon_{H}(-\grave{\boldsymbol{\Delta}})_{-\boldsymbol{\xi}}^{-\frac{2 H+d}{4}} \phi\right) & & \text { by definition; } \\
& =\exp \left(-\frac{1}{2} \epsilon_{H}^{2}\left\|(-\grave{\boldsymbol{\Delta}})_{-\boldsymbol{\xi}}^{-\frac{2 H+d}{4}} \phi\right\|^{2}\right) & & \text { by (8). }
\end{array}
$$

The above form is positive-definite and continuous, as a consequence of the continuity of the left inverse and the positive-definiteness and continuity of $Z_{W}$. The BochnerMinlos theorem therefore applies to it.

All of the properties of vector $\mathrm{fBm}$ listed in the next subsection can be deduced from the formula we have just derived.

\subsection{Some properties of vector $\mathrm{fBm}$}

In this subsection we briefly list some of the main properties of vector $\mathrm{fBm}$. Proofs have been omitted due to space limitations. They will appear in a separate publication [21].

Self-similarity: $\boldsymbol{B}_{H, \boldsymbol{\xi}}(\sigma \boldsymbol{x})$ has the same statistics as the field $\sigma^{H} \boldsymbol{B}_{H, \boldsymbol{\xi}}(\boldsymbol{x})$. In other words, the random field behaves like a stochastic fractal. This is a consequence of the scale-invariance of the left inverse operator. 
Rotation-invariance: $\boldsymbol{B}_{H, \boldsymbol{\xi}}$ interacts with rotations of the coordinate system in the way that is expected of vector quantities; i.e., for any rotation matrix $\Omega$, the random fields $\boldsymbol{B}_{H, \boldsymbol{\xi}}(\boldsymbol{x})$ and $\boldsymbol{\Omega} \boldsymbol{B}_{H, \boldsymbol{\xi}}\left(\boldsymbol{\Omega}^{\top} \boldsymbol{x}\right)$ have the same stochastic character. This, again, follows from the rotation-invariance of the left inverse.

Non-stationarity and stationary increments: As in the scalar case, vector $\mathrm{fBm}$ is non-stationary, but has stationary increments for $0<H<1$ in the sense that for any step size $\boldsymbol{h}$, the increment field

$$
\boldsymbol{L}_{\boldsymbol{h}}(\boldsymbol{x}):=\boldsymbol{B}_{H, \boldsymbol{\xi}}(\boldsymbol{x}+\boldsymbol{h})-\boldsymbol{B}_{H, \boldsymbol{\xi}}(\boldsymbol{x})
$$

is stationary. More generally, for $H>1$, the increments of order $\lfloor H\rfloor+1$ are stationary.

Covariance structure: The covariance matrix of the increment $\boldsymbol{B}_{H, \boldsymbol{\xi}}(\boldsymbol{x})-\boldsymbol{B}_{H, \boldsymbol{\xi}}(\boldsymbol{y})$ (also known as the variogram) is given by the relation

$$
\begin{array}{r}
\mathbb{E}\left\{\left[\boldsymbol{B}_{H, \boldsymbol{\xi}}(\boldsymbol{x})-\boldsymbol{B}_{H, \boldsymbol{\xi}}(\boldsymbol{y})\right]\left[\boldsymbol{B}_{H, \boldsymbol{\xi}}(\boldsymbol{x})-\boldsymbol{B}_{H, \boldsymbol{\xi}}(\boldsymbol{y})\right]^{\mathrm{\top}}\right\} \\
=2 \boldsymbol{\Phi}_{\left(\eta_{1}, \eta_{2}\right)}^{H}(\boldsymbol{x}-\boldsymbol{y})
\end{array}
$$

with

$$
\begin{aligned}
& \eta_{1}=\log \left(\frac{2 H+1}{2 H+d} \mathrm{e}^{-2 \xi_{1}}+\frac{d-1}{2 H+d} \mathrm{e}^{-2 \xi_{2}}\right), \\
& \eta_{2}=\log \left(\frac{1}{2 H+d} \mathrm{e}^{-2 \xi_{1}}+\frac{2 H+d-1}{2 H+d} \mathrm{e}^{-2 \xi_{2}}\right),
\end{aligned}
$$

with $\boldsymbol{\Phi}_{\left(\eta_{1}, \eta_{2}\right)}^{H}$ denoting the matrix-valued function defined in Eqn (2). In the case of $\boldsymbol{\xi}=\mathbf{0}$ the matrix $\boldsymbol{\Phi}_{\eta}^{H}$ becomes diagonal. Consequently, the trivial multi-component extension of $\mathrm{fBm}$ as a vector of independent scalar $\mathrm{fBm}$ fields is included in our formulation as a special case.

Fractional calculus: The stochastic vector field $\boldsymbol{B}_{H, \boldsymbol{\xi}}$ is whitened by the generalized fractional Laplacian operator $(-\boldsymbol{\Delta})_{\xi}^{\frac{H}{2}+\frac{d}{4}}$. In addition, for positive $H$ and $H^{\prime}$ with $0<H+H^{\prime}<1$ and for any $\boldsymbol{\xi}$ and $\xi^{\prime} \in \mathbb{R}^{2}$ we have

$$
(-\boldsymbol{\Delta})_{\boldsymbol{\xi}^{\prime}}^{H^{\prime}} \boldsymbol{B}_{H+H^{\prime}, \boldsymbol{\xi}+\boldsymbol{\xi}^{\prime}}=\boldsymbol{B}_{H, \boldsymbol{\xi}}
$$

and

$$
\boldsymbol{B}_{H+H^{\prime}, \boldsymbol{\xi}+\boldsymbol{\xi}^{\prime}}=(-\dot{\boldsymbol{\Delta}})_{-\boldsymbol{\xi}^{\prime}}^{-H^{\prime}} \boldsymbol{B}_{H, \boldsymbol{\xi}}
$$

The above results mean that suitable forms of fractional integration and differentiation map a given fractional Brownian vector field to another $\mathrm{fBm}$ field with modified parameters.

Irrotational and solenoidal behaviour: With the aid of dual operators and in keeping with the factorization property noted in $\$ 2.2$, it is possible to show that as $\xi_{1}-\xi_{2} \rightarrow \infty, \boldsymbol{B}_{H, \boldsymbol{\xi}}$ becomes divergencefree (solenoidal). Similarly, as $\xi_{1}-\xi_{2} \rightarrow-\infty, \boldsymbol{B}_{H, \boldsymbol{\xi}}$ exhibits curl-free (irrotational) behaviour. These extreme cases mark the range of directional comportment exhibited by these models.

\section{SIMULATION}

It is possible to compute the joint distribution of the product of $\boldsymbol{B}_{H, \boldsymbol{\xi}}$ with a family of test functions $\left\{\boldsymbol{\phi}_{i}\right\}$ by finding the inverse Fourier transform of $Z_{\boldsymbol{B}_{H, \boldsymbol{\xi}}}\left(\sum_{i} \omega_{i} \boldsymbol{\phi}_{i}\right)$. Or, in the spatial domain, one can use the fact that

$$
\begin{aligned}
\left\langle\boldsymbol{B}_{H, \boldsymbol{\xi}}, \boldsymbol{\phi}\right\rangle & =\epsilon_{H}\left\langle(-\dot{\boldsymbol{\Delta}})_{-\boldsymbol{\xi}}^{-H / 2-d / 4} \boldsymbol{W}, \boldsymbol{\phi}\right\rangle \\
& =\epsilon_{H}\left\langle\boldsymbol{W},(-\grave{\boldsymbol{\Delta}})_{-\boldsymbol{\xi}}^{-H / 2-d / 4} \boldsymbol{\phi}\right\rangle
\end{aligned}
$$

to compute the distribution of samples of $\boldsymbol{B}_{H, \boldsymbol{\xi}}$ [22].

A simpler but less rigorous approach to simulation is to apply Eqns (7) and (3) in the Fourier domain on discrete white Gaussian noise. Using this approach, we generated instances of the output in 2D that are displayed in the following figures (Figs 1a-c). The visualization technique used in the figures consists in directional smoothing of a noise image in the direction of flow, in what is known as line integral convolution (LIC) visualization (we used Mathematica's implementation).

\section{CONCLUSION}

In this contribution we introduced generic stochastic models for self-similar vector phenomena that form a family of non-stationary Gaussian random vector fields. The construction was guided by requiring the model to satisfy certain invariance properties. The choice of these invariance properties was motivated on the basis of two considerations. The first related to self-similarity and fractality - qualities that have led to stochastic models with applications in different areas of science and engineering. The second type of invariances we considered were derived from the laws of transformation of physical vectors.

After defining the extended family of fractional Brownian motion vector fields by means of their characteristic functional, we listed some of their main properties that can be of interest both from a theoretical point of view and in stochastic modelling applications. In particular, it is important to emphasize that these new models can exhibit a full range of vectorial behaviour, from completely irrotational (curl-free) to fully solenoidal (divergence-free). Such versatility suggests that these random vector fields could be suitable candidates for modelling vector data arising in different fields of application such as fluid dynamics, field theory, and imaging science. The theoretical study of these models can also be of independent interest to probability and information theorists, as has been the case with their 


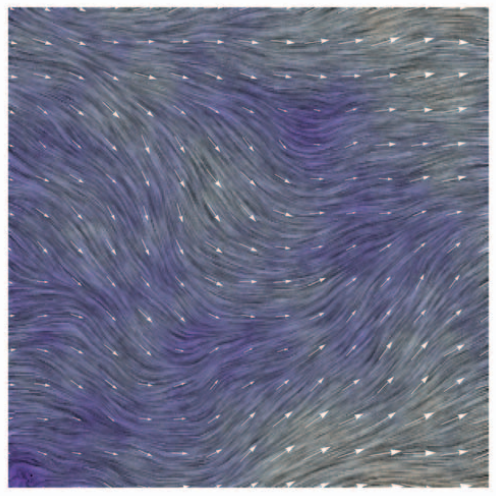

(a) $H=0.60, \xi_{1}=0, \xi_{2}=0$ (LIC)

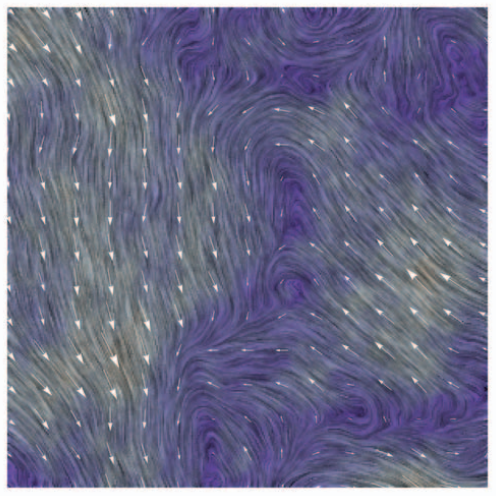

(b) $H=0.60, \xi_{1}=100, \xi_{2}=0$ (LIC)

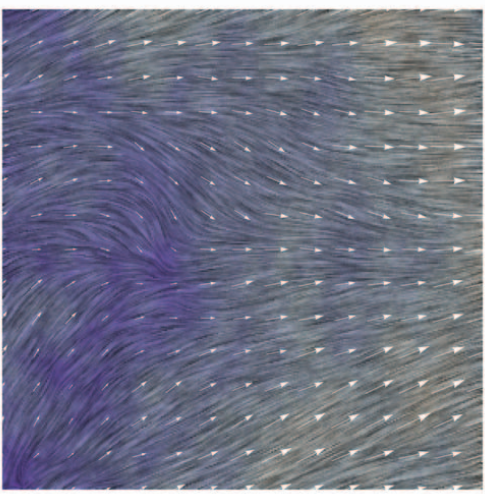

(c) $H=0.60, \xi_{1}=0, \xi_{2}=100(\mathrm{LIC})$

Fig. 1: Examples of synthesized vector fractional Brownian motion for $H=0.60$ and different values of $\xi_{1}$ and $\xi_{2}$. The vector field has been visualized using line integral convolution (see text) with local amplitudes inversely coded by saturation levels. The effect of parameters $\xi_{1}$ and $\xi_{2}$ on the directional behaviour of the field is clearly visible.

predecessors (scalar Brownian and fractional Brownian processes and fields) in the past.

\section{REFERENCES}

[1] A. N. Kolmogoroff, “Wienersche Spiralen und einige andere interessante Kurven im Hilbertschen Raum," C. R. (Doklady) Acad. Sci. URSS, vol. 26, no. 2, pp. 115-118, 1940.

[2] B. B. Mandelbrot and J. W. Van Ness, "Fractional Brownian motions, fractional noises and applications," SIAM Rev., vol. 10, no. 4, pp. 422-437, 1968.

[3] B. B. Mandelbrot, Gaussian Self-Affinity and Fractals: Globality, The Earth, $1 / f$ Noise, and $R / S$, ser. Selecta (Old or New). New York: Springer, 2002, vol. H.

[4] M. S. Keshner, " $1 / f$ noise," Proc. IEEE, vol. 70, no. 3, pp. 212-218, March 1982.

[5] W. E. Leland, W. Willinger, M. S. Taqqu, and D. V. Wilson, "On the self-similar nature of ethernet traffic," SIGCOMM Comput. Commun. Rev., vol. 25, no. 1, pp. 202-213, 1995.

[6] M. S. Taqqu, V. Teverovsky, and W. Willinger, "Is network traffic self-similar or multifractal?" Fractals, vol. 5, no. 1, pp. 63-73, March 1997.

[7] B. Pesquet-Popescu and J. Lévy Véhel, "Stochastic fractal models for image processing," IEEE Signal Processing Mag., vol. 19, no. 5, pp. 48-62, 2002.

[8] A. P. Pentland, "Fractal-based description of natural scenes," IEEE Trans. Pattern Anal. Machine Intell., vol. PAMI-6, no. 6, pp. 661-674, 1984.

[9] N. Sarshar and X. Wu, "On rate-distortion models for natural images and wavelet coding performance," IEEE Trans. Image Processing, vol. 16, no. 5, pp. 1383-1394, May 2007.

[10] P. Lévy, Le mouvement brownien, ser. Mémorial des sciences mathématiques. Paris: Imprimerie Gautheir-Villars, 1954, vol. Fascicule CXXVI, publié sous le patronage de l'Académie des sciences de Paris.

[11] J.-P. Kahane, Some Random Series of Functions, 2nd ed. Cambridge University Press, 1985.
[12] P. D. Tafti, D. Van De Ville, and M. Unser, "Invariances, Laplacian-like wavelet bases, and the whitening of fractal processes," IEEE Trans. Image Processing, vol. 18, no. 4, pp. 689-702, April 2009.

[13] A. Benassi, S. Jaffard, and D. Roux, "Elliptic gaussian random processes," Rev. Mat. Iberoamericana, vol. 13, no. 1, pp. 19-90, 1997.

[14] I. M. Gel'fand and N. Y. Vilenkin, Applications of Harmonic Analysis, ser. Generalized Functions. Academic Press, 1964, vol. IV, translated by Amiel Feinstein.

[15] A. N. Kolmogorov, "A note on the papers of R. A. Minlos and V.Sazonov," Theory Probab. Appl., vol. 4, pp. 221-223, 1959.

[16] G. Samorodnitsky and M. S. Taqqu, Stable Non-Gaussian Random Processes: Stochastic Models with Infinite Variance. New York: Chapman and Hall, 1994.

[17] R. L. Dobrushin, "Gaussian and their subordinated selfsimilar random generalized fields," Ann. Probab., vol. 7, no. 1, pp. 1-28, 1979.

[18] A. F. Stalder, M. F. Russe, A. Frydrychowicz, J. Bock, J. Hennig, and M.Markl, "Quantitative 2D and 3D phase contrast MRI: Optimized analysis of blood flow and vessel wall parameters," Magn. Reson. Med., vol. 60, no. 5, pp. 1218-1231, 2008.

[19] M. Farge, N. Kevlahan, V. Perrier, and E. Goirand, "Wavelets and turbulence," Proc. IEEE, vol. 84, no. 4, pp. 639-669, Apr 1996.

[20] M. Arigovindan, "Variational reconstruction of vector and scalar images from non-uniform samples," Ph.D. dissertation, EPFL, Biomedical Imaging Group, 2005.

[21] P. D. Tafti and M. Unser, "Fractional Brownian vector fields," submitted.

[22] — " "Self-similar random vector fields and their wavelet analysis," in Proc. SPIE Conference on Mathematical Imaging: Wavelets XIII, vol. 7446, 74460Y, San Diego CA, USA, August 2009, pp. 74 460Y-1-74 460Y-8. 